\title{
Informationen der SAMM
}

Manuelle Medizin 2016 · 54:362 DOI 10.1007/s00337-016-0181-0

(c) Springer-Verlag

Berlin Heidelberg 2016

\section{$\nabla_{\Delta} \nabla_{\Delta} \nabla_{\Delta} \nabla \mathbf{V}$ \\ MANUELLEMEDIZIN \\ SAMM}

\section{Schweizerische Ärztegesellschaft für Manuelle Medizin SAMM}

Fortbildung in Manueller Medizin

Anmeldung und Auskünfte:

Sekretariat SAMM

Kolumbanstrasse 2

9008 St.Gallen

Tel. 0712465181

Fax. 0712465101

info@samm.ch

V.i.S.d.P.

Dr. rer. publ. HSG Sven Bradke

\section{Kongress für Manuelle Medizin}

\section{Jahreskongress SAMM 2016}

24.-26. November 2016, Interlaken

zum Thema "Cervicothorakaler-Übergang/Brustwirbelsäule

(CTÜ/BWS)"

SAMM GV

25. November 2016, Interlaken, $12.00 \mathrm{Uhr}$

\section{FM1 2017}

07.-08. April 2017

Fortbildungsmodul Mobilisation ohne Impuls

Centre Löwenberg, Murten

FM2 2017

07.-08. April 2017

Fortbildungsmodul Mobilisation mit Impuls

Centre Löwenberg, Murten

Anmeldungen FM1 und FM2 an info@samm.ch

Kurskosten

Inkl. Unterkunft CHF 905,-

Exkl. Unterkunft CHF 800,-

Abmeldebedingungen

30 Tage vor Kursbeginn $50 \%$ der Kurskosten

12 Tage vor Kursbeginn $80 \%$ der Kurskosten

6 Tage vor Kursbeginn $100 \%$ der Kurskosten

\section{Fortbildungsangebot 2017}

Kursinformationen und Daten folgen. 